\title{
NOUVELLe
}

\section{Réparation du cortex adulte lésé par transplantation de neurones embryonnaires}

Afsaneh Gaillard, Mohamed Jaber
Institut de Physiologie et Biologie Cellulaires,

Université de Poitiers, CNRS,

40, avenue du Recteur Pineau, 86022 Poitiers, France.

afsaneh.gaillard@univ-poitiers.fr
> La perte des neurones corticaux est une caractéristique commune à de nombreuses conditions neuropathologiques comme les lésions (traumatiques, vasculaires...) ou les maladies neurodégénératives (sclérose latérale amyotrophique, maladies de Huntington, d'Alzheimer...). La repousse axonale joue un rôle important dans la récupération fonctionnelle des circuits moteurs endommagés. La plus longue projection axonale dans le cerveau des mammifères a pour origine les neurones pyramidaux du cortex moteur qui projettent au niveau des motoneurones et des interneurones de la corne ventrale de la moelle épinière. La dégénérescence de ces axones provoque la mort des neurones pyramidaux, ce qui est à l'origine des troubles de la motricité. Le remplacement des projections cortico sous-corticales et plus particulièrement des projections corticospinales est un facteur critique de récupération de fonctions motrices [1].

La technique de neurotransplantation est un outil précieux, notamment dans le domaine de la recherche clinique, où elle représente un potentiel thérapeutique non négligeable pour traiter diverses atteintes neurologiques. La transplantation intracérébrale de cellules embryonnaires dans des modèles animaux de pathologies liées à une perte de neurones est devenue une approche thérapeutique pour la reconstruction des circuits endommagés.

Très peu d'études ont été consacrées à la possibilité de la repousse axonale de cellules embryonnaires corticales transplantées dans le cortex moteur adulte lésé. De plus, les résultats obtenus dans les expériences testant la possibilité d'une telle repousse axonale sont sujets à contro- verse. En effet, il a été montré dans certains cas une absence de projections [2], des projections éparses et, dans les cas les plus favorables, des projections modérées et à très faible distance du transplant [3-5]. Aucune, parmi les études citées cidessus, n'indique un rétablissement pointà-point des circuits moteurs endommagés en réponse à une greffe de cellules embryonnaires. Ces différences peuvent être imputables aux contraintes méthodologiques imposées par la technique d'injection dans le transplant, pour visualiser des fibres émanant du greffon. Dans les études citées ci-dessus, des neurotraceurs antérogrades ont été injectés au sein du transplant. Cependant, le recours à des marqueurs antérogrades permet seulement de visualiser une faible partie des projections. En effet, il est nécessaire de limiter la taille du point d'injection pour éviter que le neurotraceur ne diffuse dans le tissu hôte. Par voie de conséquence, peu de cellules du transplant captent le neurotraceur.

\section{Croissance axonale après}

la greffe de neurones embryonnaires

Nous avons dernièrement démontré les capacités inattendues de développement axonal de neurones immatures transplantés dans le système nerveux central (SNC) adulte [6]. En effet, afin de tester la possibilité de la reconstruction des voies motrices endommagées, chez la souris adulte, nous avons transplanté un fragment de tissu embryonnaire (દ14) cortical, contenant des neurones immatures et d'autres types de cellules comme les cellules gliales, issu de souris transgéniques exprimant une protéine fluorescente, la green fluorescent protein (GFP) [7], au sein du cortex moteur, préalablement lésé par aspiration. L'utilisation de souris transgéniques dont les cellules expriment la GFP permet de distinguer le greffon de l'hôte et de suivre les connexions des neurones transplantés au niveau de leurs cibles. Avec cette nouvelle approche expérimentale, nous avons montré que des neurones embryonnaires issus du cortex moteur et transplantés dans un site homotypique lésé chez un receveur adulte, développent des projections vers les cibles normales du site cortical dans lequel ils sont greffés. En effet, des fibres et terminaisons sont observées systématiquement dans le cortex ipsi- et controlatéral, par rapport au site de transplantation (Figure $I A-D$ ) ainsi que dans le striatum (Figure $I B-D)$. Dans certains cas, le thalamus (Figure ID), le noyau du pont (Figure IF), et la moelle épinière (Figure $1 \mathrm{H}$ ), contiennent également des fibres marquées. Nous avons montré également qu'une partie de ces projections est myélinisée. De plus les neurones embryonnaires sont bien intégrés dans les circuits de l'hôte car les axones de l'hôte établissent des contacts synaptiques avec les neurones transplantés et les axones des neurones transplantés établissent des contacts synaptiques avec les neurones de l'hôte. Nous avons aussi réalisé les contrôles nécessaires afin de vérifier que le signal GFP détecté au sein des régions cibles du cortex moteur ne résulte pas d'une fusion des cellules transplantées $\mathrm{GFP}^{+}$avec les cellules intactes de l'hôte qui auraient pu transporter la protéine GFP vers les régions cibles.

Ces résultats montrent pour la première fois que le système nerveux central adulte 


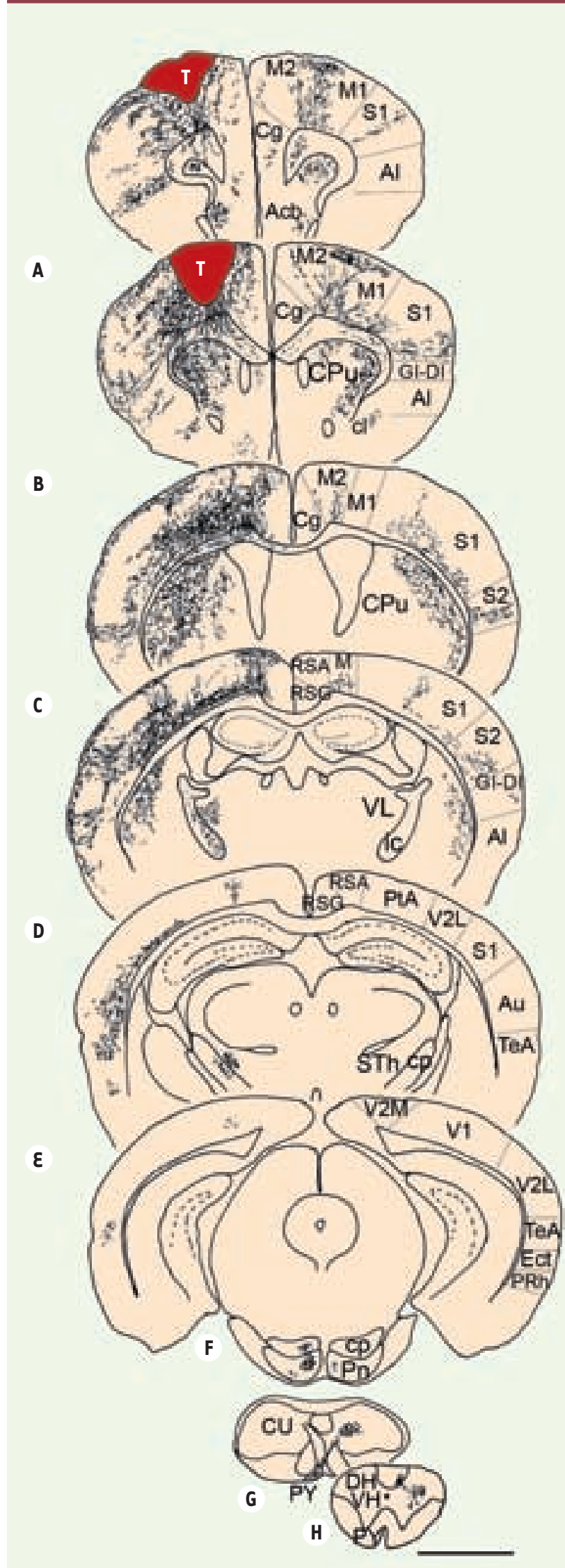

permet la repousse axonale car des neurones embryonnaires transplantés sont capables d'étendre leurs prolongements vers les cibles appropriées du cortex moteur de l'hôte. Ainsi, la mise en évidence des potentialités de développement axonal de neurones embryonnaires transplantés chez un receveur adulte permet d'envisager la reconstruction de voies nerveuses centrales lésées. De plus, nous avons montré que des neurones embryonnaires issus d'autres régions corticales que le cortex moteur sont incapables de réparer des voies motrices endommagées. $\varepsilon n$ effet, des neurones embryonnaires du cortex visuel transplantés dans le cortex moteur adulte préalablement lésé projettent vers les cibles du cortex visuel et non pas vers les cibles du cortex moteur.

Dans ce cadre, les recherches en cours sur l'intérêt des cellules souches pour la transplantation devraient se focaliser sur les facteurs et les signaux, qui restent à identifier, et qui non seulement vont promouvoir la maturation des cellules souches en neurones mais surtout en un phénotype neuronal spécifique et approprié en fonction de la région à réparer. Sans cela, le risque est potentiellement important d'obtenir des projections non spécifiques et aberrantes.

\section{Quelles perspectives}

pour le traitement de la maladie de Parkinson?

Ce dernier point est particulièrement crucial étant donné le nombre substantiel de travaux en cours dans le cadre de stratégies réparatrices de la maladie de Parkinson où des efforts significatifs sont déployés afin de restaurer des niveaux appropriés de dopamine striatale par transplantation de neurones dopaminergiques issus de cellules souches. Dans ce cadre, si, comme le montrent nos résultats, des neurones transplantés à distance des sites de projections sont capables d'envoyer des projections à longue distance vers leurs cibles, on peut envisager transplanter des cellules thérapeutiques au sein de la substance noire, la région du cerveau touchée par la mort de neurones dopaminergiques, plutôt qu'au sein du striatum, la région cible de ces neurones où sont pratiquées les greffes actuel-

Figure 1. Représentation schématique de coupes frontales ordonnées selon l'axe rostrocaudal. Les panneaux $\boldsymbol{A}$ à $\boldsymbol{H}$ illustrent la distribution des fibres $\mathrm{GFP}^{+}, 60$ jours après greffe. Le transplant développe un ensemble de projections vers la plupart des cibles corticales et sous-corticales qui sont normalement contactées par les neurones du cortex moteur. Échelle : $600 \mu \mathrm{m}$. cc: corpus callosum; cp: cerebral pedoncule; CPu: caudate putamen; dcs : dorsal corticospinal tract; ic: internal capsule; $\mathrm{M}$ : motor cortex; $\mathrm{Pn}$ : pontine nuclei; Py: pyramidal tract; S: sensorimotor cortex; Sth: subthalamic nucleus; $\mathrm{T}$ : transplant; VL: ventrolateral thalamic nucleus. 
lement. La transplantation nigrale plutôt que striatale présenterait de nombreux avantages comme la restauration des circuits des ganglions de la base, un possible effet trophique des cellules greffées sur les cellules dopaminergiques restantes de l'hôte et une meilleure intégration du greffon au sein de sa structure de destination naturelle, la substance noire.

De plus, nos résultats, en accord avec ceux d'autres groupes $[8,9]$ suggèrent que tous les neurones dopaminergiques ne seraient pas capables de ré-innerver le striatum et qu'il faudrait induire la différenciation des cellules souches qui pourraient être utilisées comme source de neurones en neurones dopaminergiques de type nigral. Toutes ces questions constituent les défis de demain dans le domaine de la réparation par des cellules embryonnaires des voies lésées, et plus tard, par les cellules souches dont la différenciation a été maîtrisée et orientée vers les phénotypes précis requis [10-12]. $\diamond$

Repair of damaged adult cortex by

transplantation of embryonic neurons

\section{REMERCIEMENTS}

les travaux détaillés ici ont été soutenus par la Fondation de l'Avenir.

\section{RéFÉRENCES}

1. Emsley JG, Mitchell BD, Magavi SSP, et al. The repair of complex neuronal circuitry by transplanted and endogenous precursors. NeuroRx $2004 ; 1: 452-71$.

2. Gonzalez MF, Sharp FR, Loken JE. Fetal frontal cortex transplanted to injured motor/sensory cortex of adult rats: reciprocal connections with host thalamus demonstrated with WGA-HRP. Exp Neurol 1998 ; 99: 154-65.

3. Isacson 0 , Wictorin K, Fischer W, et al. Fetal cortical cell suspension grafts to the excitotoxically lesioned neocortex: anatomical and neurochemical studies of trophic interactions. Prog Brain Res 1988; 78 : 13-26.

4. Sorensen JC, Grabowski M, Zimmer J, Johansson BB. Fetal neocortical tissue blocks implanted in brain infarcts of adult rats interconnect with the host brain. Exp Neurol 1996; 138 : 227-35.
5. Guitet J, Garnier C, Ebrahimi-Gaillard A, Roger M. Efferents of frontal or occipital cortex grafted into adult rat's motor cortex. Neurosci Lett 1994 ; $180: 265-8$.

6. Gaillard A, Prestoz L, Dumartin B, et al. Reestablishment of damaged adult motor pathways by grafted embryonic cortical neurons. Nat Neurosci 2007 ; 10 : 1294-9.

7. Okabe M, Ikawa M, Kominami K, et al. Green mice as a source of ubiquitous green cells. FEBS Lett 1997 ; 407: 313-9.

8. Bjorklund LM, Sanchez-Pernaute R, Chung S, et al. Embryonic stem cells develop into functional dopaminergic neurons after transplantation in a Parkinson rat model. Proc Natl Acad Sci USA 2002; 99: 2344-9.

9. Thompson L, Barraud P, Andersson $\varepsilon$, et al. Identification of dopaminergic neurons of nigral and ventral tegmental area subtypes in grafts of fetal ventral mesencephalon based on cell morphology, protein expression, and efferent projections. J Neurosci 2005 ; 27 : 6467-77.

10. Fluckiger AC, Dehay C, Savatier P. Cellules souches embryonnaires et thérapies cellulaires du système nerveux. Med Sci (Paris) 2003 ; 19: 699-708.

11. Perrier AL. Des cellules souches embryonnaires humaines pour le traitement de la maladie de Parkinson? Med Sci (Paris) 2005 ; 21 : 11-3.

12. Cazillis $M$, Lelièvre V, Gressens $P$. Différenciation neurale des cellules souches embryonnaires. Med Sci (Paris) $2005 ; 21: 484-90$.

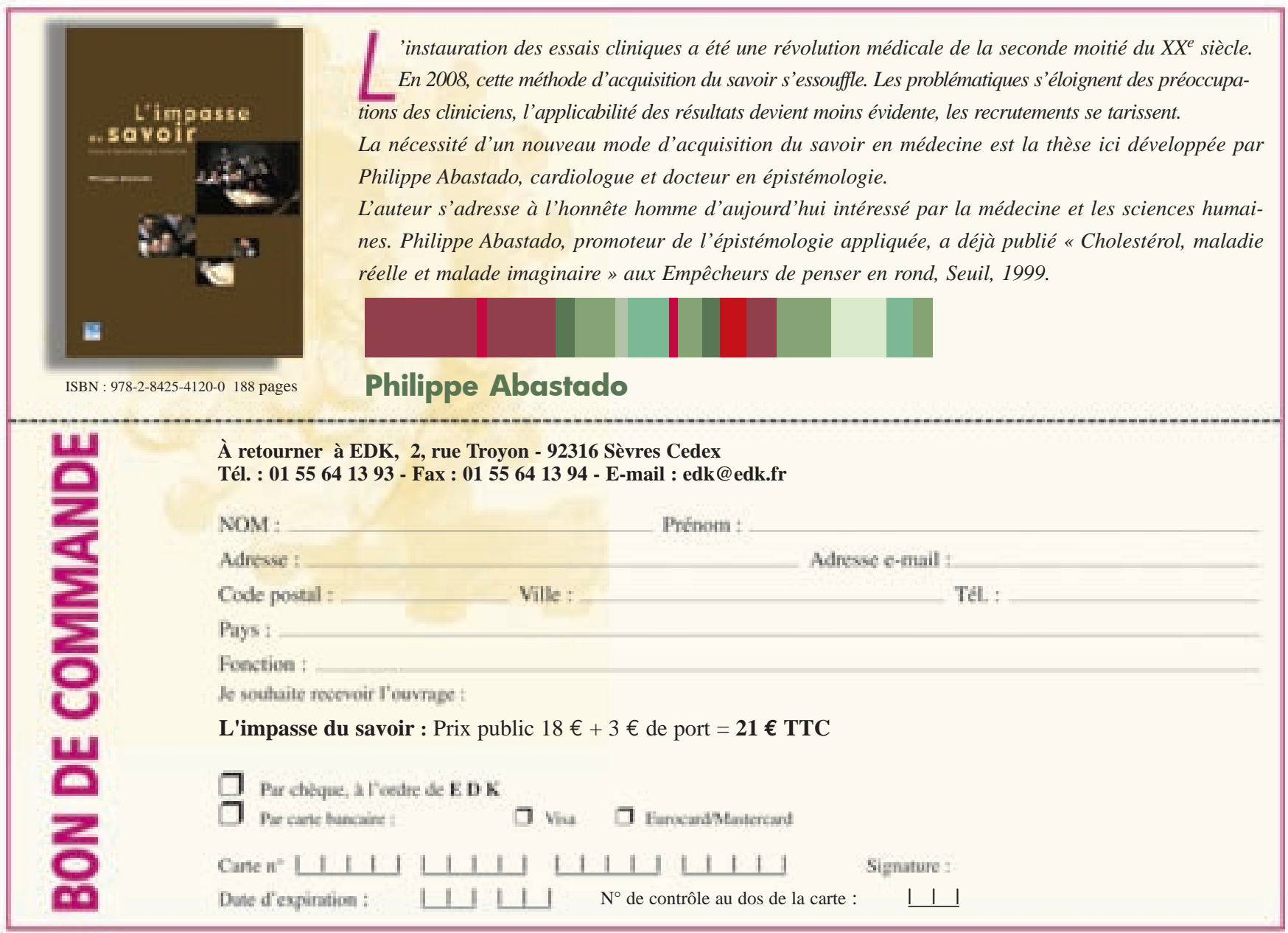

